\title{
Image Retrieval Framework for Medical Images using Improved SVM
}

\author{
Savitri Chandra ${ }^{1}$, Latika Pinjarkar ${ }^{2}$ \\ M. Tech Scholar, Computer Science \& Engg Dept. Shankaracharya Group of Institutions, Bhilai (C.G.), India ${ }^{1}$ \\ Assoc. Prof. Computer Science \& Engg Dept. Shankaracharya Group of Institutions, Bhilai (C.G.), India ${ }^{2}$
}

\begin{abstract}
Extraction of certain features from the images is quite difficult to conclude as a result, which can be further used for some kind of specific purpose. In medical field, there is a huge amount of digital images that are related with different diseases. Since, it is a field that demands keen observation of this digital data and responsible work from the concern staff; the chances of error are also very high. This project is an attempt to assistant the medical staff, especially in case of brain tumor to draw a more accurate conclusion on the basis of pattern matching of the captured image with the pre-designed training set. The primary objective of this project is to collaborate the latest available technologies with the knowledge set of the experts to work in a more accurate way. It also aims to serve the man-kind and assist the surgeons in their noble work.
\end{abstract}

Keywords: Content Based Image Retrieval (CBIR), Query by image content (QBIC), Content-based visual information retrieval (CBVIR), Feature Extraction, Low Level Feature extraction.

\section{INTRODUCTION}

Content-based image retrieval (CBIR), also known as query by image content (QBIC) and content-based visual information retrieval (CBVIR) is the application of computer vision techniques to the image retrieval problem, that is, the problem of searching for digital images in large databases Content based search analyses the contents of the image rather than the metadata such as keywords, tags, or descriptions associated with the image. The term "content" in this context might refer to colours, shapes, textures, or any other information that can be derived from the image itself [1]. The architecture of a CBIR system can be understood as a basic set of modules that interact within each other to retrieve the database images according to a given query. To retrieve images, users provide the retrieval system with query images or sketched figures. The system then changes the query image into its internal representation of feature vectors. The similarities/differences between the feature vectors of the query example and those of the images in the database are then calculated and retrieval is performed with the aid of an indexing scheme [2]. Research and development issues in CBIR cover a range of topics, many shared with mainstream image processing and information retrieval [3]. Some of the most important are:

- understanding image users' needs and information-seeking behaviour

- identification of suitable ways of describing image content

- extracting such features from raw images

- providing compact storage for large image databases

- matching query and stored images in a way that reflects human similarity judgements

- efficiently accessing stored images by content

- providing usable human interfaces to CBIR systems

Major application areas of CBIR include:-

A. Crime Prevention-The Law enforcement agencies typically maintain large archives of visual evidence, including past suspects' facial photographs (generally known as mug shots), fin whenever a serious crime is committed, they can compare evidence from the scene of the crime for its similarity to records in their archives [4].

B. The Military- Military applications of imaging technology are Recognition of enemy aircraft from radar screens, identification of targets from satellite photographs, and provision of guidance systems for cruise missiles [4].

C. Medical Diagnosis-the prime requirement for medical imaging systems is to be able to display images relating to a named patient, there is increasing interest in the use of CBIR techniques to aid diagnosis by identifying similar past cases[4].

Basically CBIR systems use two approaches for retrieving the images from the image data base which are:

A. Text-based approach: Text based method used the keywords descriptions as a input and get the desired output 


\section{ISO 3297:2007 Certified}

Vol. 5, Issue 7, July 2017

in the form of similar types of images [5]. It applies traditional text retrieval techniques to image annotations or descriptions. Most of the image retrieval systems are text-based, but images frequently have little or no accompanying textual information. Keywords are words or phrases that are described content. They can be used as metadata to describe images, text documents, database records, and Web pages.

B. Content-Based Approach: Content based approach using image as an input query and it generate the output of similar types of images [5]. The term "Content based" means that it will search the actual content of an image. Information retrieval means the process of converting a request for information into a meaningful set of reference. CBIR is a technology that in principle helps organize digital image archives according to their visual content. This system distinguishes the different regions present in an image based on their similarity in colour, texture, shape, etc. and decides the similarity between two images by reckoning the closeness of these different regions [6].

Feature extraction plays an important part in the fields of pattern recognition and data mining technology. It basically extracts the meaningful feature subset from original sets by some rules. It is done, however, to reduce the complexity of space and the time of machine training and, also to achieve the goal of dimensionality reduction [7].

The techniques for feature extraction can be divided into following four categories:

- Non transformed structural characteristic includes moments, model parameters, and power and phase information.

- A transformed structural characteristic includes frequency spectra and subspace mapping methods. A structural description includes parsing techniques, formal languages and their grammars, and string matching techniques.

- Graph descriptors include semantic networks, attributed graphs and relational graphs.

Low level features of any image are as mentioned below:

A. Colour Feature: Colour is one of the important features that make possible the recognition of images by humans. It is a property which is dependent on the reflection of light to the eye and also the processing of that information by the brain.

B. Texture Feature: Texture is one of the useful characterizations for a wide variety of images. It is generally believed that for recognition and interpretation, human visual systems use texture. In general, it is said that colour is usually a pixel property whereas texture can only be calculated from a group of pixels. There are a large number of techniques that have been proposed to extract texture features.

\section{RELATED WORK}

According to Mohanapriya S and Vadivel M [8] in their work entitled "Automatic retrival of mri brain image using multiqueries system" proposes that feature extraction plays an important role in CBIR techniques. They proposed a robust technique that concentrated on first extracting best features and then training the $\mathrm{k}-\mathrm{NN}$ classifier in order to classify and retrieve the MRI Brain Image. According to Modh Jigar S, Shah Brijesh and Shah Satish K [9] proposed a $\mathrm{K}$-Means image segmentation technique using cosine distance matrices instead of Euclidean distance in "A new kmean color image segmentation with cosine distance for satellite images". Using cosine distance matrices reduced computational time and calculation for every pixel in the image. Although colors are not frequently used in image segmentation; it gives high discriminative power to the regions present in image. Authors Ale s Proch'azka and Andrea Gavlasov'[10] in their work titled "texture segmentation and classification in biomedical image processing" proposed that feature based image segmentation is very efficient in the case of properly chosen properties defining feature vectors values associated with all image pixels. Authors Zhengrong Li , Yuee Liu, Ross Hayward and Rodney Walker [11] proposes in "empirical comparison of machine learning algorithms for image texture classification with application to vegetation management in power line corridors" proposes various machine learning algorithms and three textual features. The experimental results showed that the classification performance depends on the performance matrix, the characteristics of datasets and the feature(s) used. According to Weidong Zhao, Shuanglin Tang and Weihui Dai [12] "an improved knn algorithm based on essential vector" proposed many improved methods from different perspectives: the first method is to simplify the computation by decreasing the quantity of training samples . The second method is to find the nearest neighbor fast by introducing the fast search algorithm or establishing efficient indexing. The third method is to lower the computation complexity by decreasing the dimension of the vector space.

\section{PROPOSED METHODOLOGY}

Various types of problems may occur during the experiment within the area of Content Based Image Retrieval (CBIR) for the retrieval of the images from the database. One of several may perhaps algorithms advised KNN can be a quite potent and well-liked distinction algorithm in order to classify impression of data. Some of the problems identified are: 


\section{UGC Approved Journal}

IJIREEICE

\section{International Journal of Innovative Research in Electrical, Electronics, Instrumentation and Control Engineering}

\section{ISO 3297:2007 Certified}

Vol. 5, Issue 7, July 2017

- The main reason of the problem is no one techniques can able to give the result as the human perception or near it the previous designed system, in addition to performing linear classification.

- The problem involves entering an image query into a software application that is designed to employ Content Based Image Retrieval techniques in existing visual properties and matching them. This is done to retrieve image in the database that are visually similar to the query image.

- The text based search engines have certain limitation like inability to express query in words. For example, if we want finger print of a certain person from large collection of fingerprint images then it is impossible to express our query in terms of words, as it's cumbersome to define fingerprint of any person in words. Also, metadata is generally available in single language. Different users may express their common query with different words.

To overcome all such limitations, this scheme has been proposed. The proposed System is designed on the basis of following major steps:-

1. Collection of image database: Large numbers of digital images are stored for the designed framework contains five hundred medical images to retrieve desired medical image or images on the given as query image. These medical images can be stored in the hard disk or database. Design a database containing five hundred medical images (Brain tumor) with any one of the formats .bmp .jpg .tiff or any other image formats. The images will be gray scale image or it will be from RGB (Red, Green, Blue) color model.

2. Feature Extraction of database images: Feature Extraction is carried out by using the visible features of an image that is colors, texture and shapes. These extracted features will be stored in feature database. This phase involves extracting features of the query image and the database image. In proposed work, color based and texture based features are extracted. Color based feature extraction process is done by using three algorithms: Color autocorrelogram, HSV (Hue Saturation Value) histogram and Color moments. Texture based features are:, mean amplitude of 2D wavelet components, mean and standard deviation of wavelet coefficients. From this phase, we get two feature vectors one for the query image and another for the database images, for shape feature extraction uses moment invariant all the features are extracted and stored in feature database in the form of feature vector table.

3. Query Image: It is the image to be found in the image database where as the similar images are represent or not. Query image is Desire image which is to be retrieved from the medical image database, query image is given by the user he/she wants search the similar medical images from the medical image database.

4. Color feature Extraction of query image: One of the important features that make possible the recognition of image by human is color. Usually colors are classified in three dimensional color spaces. These could either be RBG (Red, Green and Blue), HSV(Hue Saturation, and Value) or HSB(Hue, Saturation, and Brightness).the last two are dependent on the human Perception of hue, saturation and Brightness. The proposed system uses HSV (Hue Saturation, and Value) color histogram by this image to be quantized in HSV color space

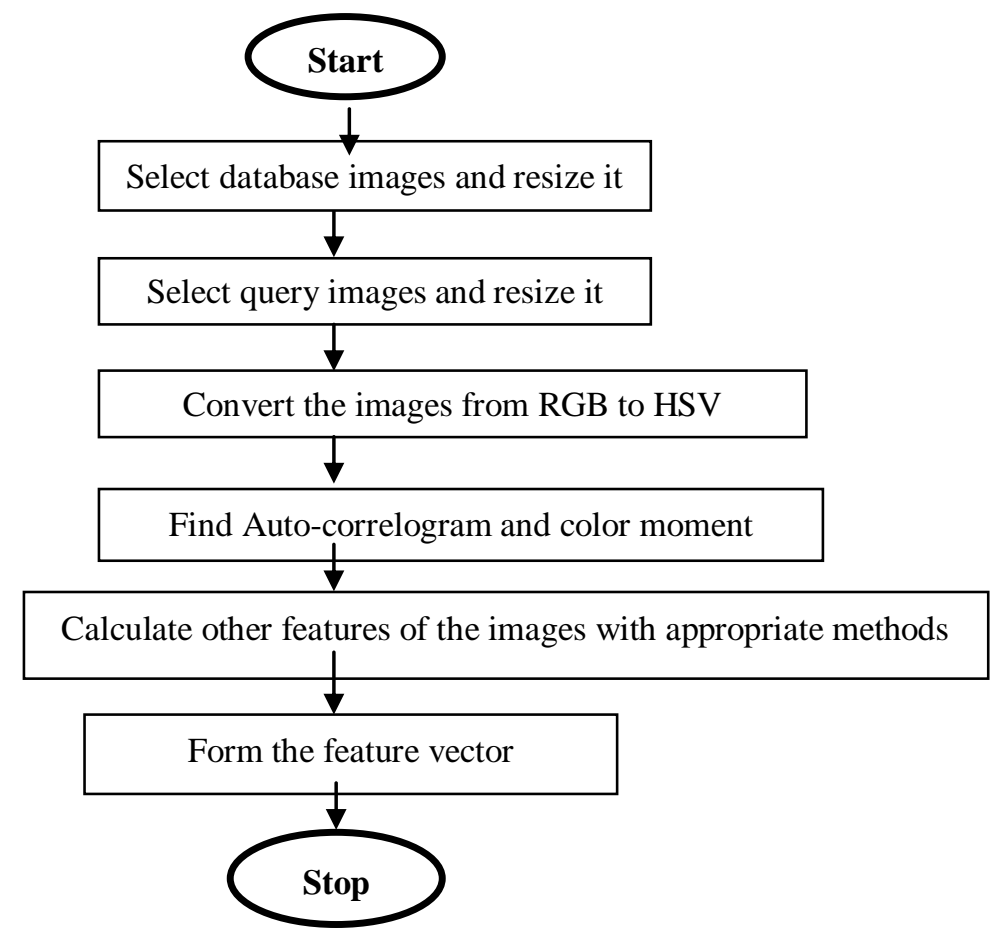

Figure 1: Flowchart for feature extraction process 
UGC Approved Journal

IJIREEICE

Vol. 5, Issue 7, July 2017

5. Similarity Measure: In this phase, features of each image stored in the database are compared to the features of the query image. Improves SVM (Support Vector Machine) is employed for classification of images based on their features. Also, similarity between the two images is measured by using euclidean distance by correlation. If the distance between feature vector of the database image and the query image is small, then that image in the database is considered as a matched image to the query image.

6. Improved SVM: The proposed work based on improved Support Vector machine has been extensively used in the CBIR system to bridge the semantic gap between low level features and human perception features. The main functions that are used to implement the improved SVM are given below because of that SVM improves the working area like represent confusion matrix and calculate the accuracy of the retrieved similar images and by using the ranking algorithm rank the image for retrieval of image. Support vector machine provides some extra i,e it is the improved SVM.

7. Retrieval of similar images: After all of the above steps system will ready to run, the image are the desired outcome of the proposed system that is retrieved similar image as we give in as input.

\section{EXPERIMENTAL RESULTS}

The performance of the framework is evaluated in terms of precision and recall. The precision is defined as the ratio of relevant images retrieved to all images retrieved, while the recall is defined as the ratio of relevant images retrieved to all relevant images in a database, or the probability given that an image is relevant that it will be retrieved. Improved SVM make confusion matrix that shows the accuracy of retrieving the desired image from the database images. Results show GUI for the image retrieval system for medical image. It shows number of expected and actual retrieved images are same. Similarity matching between the images is done by measuring Euclidean distance. Experimental results show that improved SVMs achieve significantly higher search accuracy than traditional SVMs. Once the initial validation was completed, group of images were added to the database. Then the query image was matched visually with groups of images from the database and the database images were ranked according to how similar they were perceived to be to the query. These groups serve as the expected relevant results during testing.

Precision $=$ Number of relevant images retrieved /Total number of images retrieved

Recall $=$ Number of relevant images retrieve/Total number of relevant images in the Database

Table 1: Results achieved by designed framework after performance Evaluation

\begin{tabular}{|c|c|c|c|}
\hline Names of the query image & Number of relevant images received & Precision Values & Recall values \\
\hline & 4 & 0.87 & 0.80 \\
\hline & 3 & 0.89 & 0.86 \\
\hline & 5 & & 0.84 \\
\hline \\
\hline
\end{tabular}


International Journal of Innovative Research in Electrical, Electronics, Instrumentation and Control Engineering

\section{ISO 3297:2007 Certified}

Vol. 5, Issue 7, July 2017

Table 2: Comparison of proposed approach with the referenced system

\begin{tabular}{|c|c|c|}
\hline Author & Average precision & Average Recall \\
\hline Existing Approach & 0.81 & 0.78 \\
\hline Proposed Approach & 0.85 & 0.83 \\
\hline
\end{tabular}

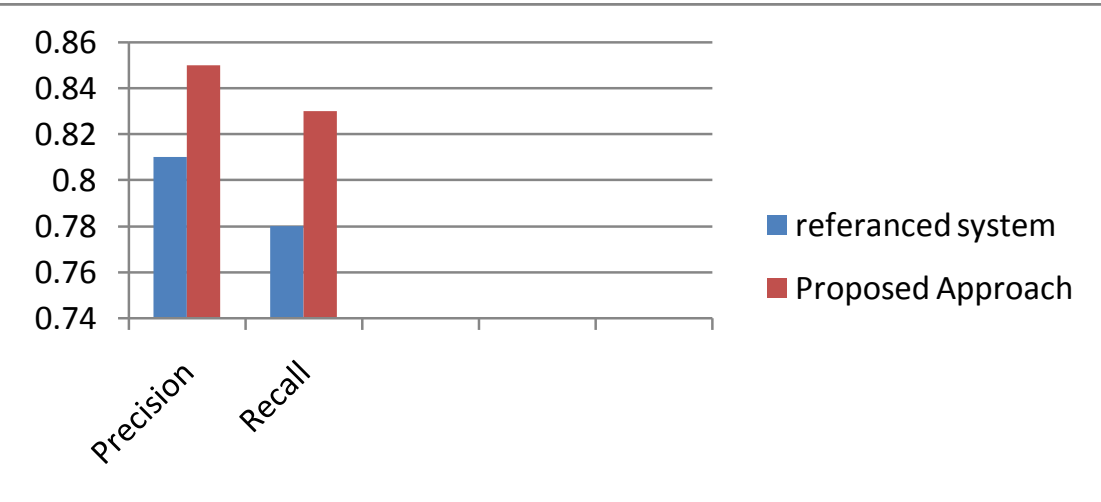

Fig.2: Average precision and recall values of proposed approach and referenced system

\section{CONCLUSION}

Our main objective of this designed framework is to retrieve the medical images from database an efficient manner by using improved Support vector machine (SVM). The number of search results depends on user; they are able to select the number of similar images that they wants to retrieve form the medical image database. Calculate the precision and recall values of designed framework and compare it from the previous referenced system and found the our proposed system provides the more efficient values then referenced system, it was done by the use of the improved SVM along with CBIR. This designed image retrieval framework using improved SVM Enhance the performance of the image retrieval for the medical images it is very flexible framework can be used for other images also. CBIR for medical images is the important application area of Content Based Image Retrieval. The proposed framework is designed using improved support vector machine technique, as a learning mechanism. The improved SVM has help to enhance the retrieval result of the proposed framework. In future this system is also implemented in the field of computer Vision which is concerned with the automated processing of images from the real world to extract and interpret information on a real time basis. In future this system is used in Astronomy to the study of celestial objects (such as stars, comets, nebulae, planets, star clusters and galaxies). Further time to retrieve the system can also be reduced in future.

\section{REFERENCES}

[1] https://en.wikipedia.org/wiki/Content-based_image_retrieval

[2] Content-based Image Retrieval: Feature Extraction Techniques and Applications , Amandeep Khokher \& Rajneesh Talwar, International Conference on Recent Advances and Future Trends in Information Technology (iRAFIT2012).

[3] http://www.leeds.ac.uk/educol/documents/00001240.htm

[4] Different Approaches of CBIR Techniques, Jagpal Singh, Jashanbir Singh Kaleka \& Reecha Sharma, International Journal of Computers \& Distributed Systems, Volume 1, Issue 2, August, 2012.

[5] A Review of Different Content Based Image Retrieval Techniques, Amit Singh, Parag Sohoni \& Manoj Kumar , International Journal of Engineering Research and General ScienceVolume 2, Issue 5, August - September 2014

[6] A Survey on Text and Content Based Image Retrieval System for Image Mining, T. Karthikeyan, P. Manikandaprabhu \& S. Nithya, International Journal of Engineering Research \& Technology (IJERT),2014

[7] Comparative Study of Different Low Level Feature Extraction Techniques, Rupali Sharma \& Er. Navdeep Singh, International Journal of Engineering Research \& Technology (IJERT) 2014

[8] Rahmani, M. K. I., Ansari, M. A., \& Goel, A. K. (2015, February). An Efficient Indexing Algorithm for CBIR. In Computational Intelligence \& Communication Technology (CICT), 2015 IEEE International Conference on (pp. 73-77). IEEE

[9] Arti Khaparde, B.L.Deekshatulu, M.Madhavilath, Zakira Farheen, Sandhya Kumari V, "Content Based Image Retrieval Using Independent Component Analysis", IJCSNS International Journal of Computer Science and Network Security, VOL.8 No.4, April 2016.

[10] Tomasz Andrysiak, Michał Chora', "ImageRetrieval Based On Hierarchical Gabor Filters, Int. J. Appl. Math. Compute. Sci., 2005, Vol. 15, No. 4, 471-480

[11] S.A.Pine, R C Patil, “Content Based Image Retrieval Using Wavelet Transform” Telecommuniction’ Thadomal Shahani Engineering College, Bandra (East), Mumbai-51.

[12] Rajakumar, K., Muttan, S.: Medical Image Retrieval using Energy Efficient Wavelet Transform. In: Second International conference on Computing, Communication and Networking Technologies, Department of Electronics and Communication Engineering College of Engineering, Guindy, Anna University, Chennai, India (2010) 